\section{Grupos de apoyo para pacientes con tuberculosis en Facebook}

\section{Support groups for patients with tuberculosis in Facebook}

\section{Sr. Editor:}

La tuberculosis es una de las principales causas de mortalidad a nivel mundial, en especial en países de bajos y medianos ingresos ${ }^{1}$. El tratamiento de esta enfermedad se basa en la administración de antibióticos durante al menos seis meses. Durante este lapso, el soporte social del paciente es importante, dado que mejora su adherencia al tratamiento y su calidad de vida²
Este soporte puede darse por medio de redes sociales virtuales, como son los grupos de Facebook. Estas redes facilitan la interacción de personas con la misma enfermedad, permitiendo que compartan dudas, experiencias y consejos; además de apoyarse y alentarse mutuamente. Estos grupos también pueden contar con profesionales de la salud para resolver dudas y verificar la veracidad de la información ${ }^{3}$.

Se han publicado experiencias positivas sobre grupos creados para Cáncer de mama ${ }^{3}$, alimentación saludable ${ }^{4}$, Diabetes y Cáncer colorrectal ${ }^{5}$.

En diciembre de 2015 realizamos una búsqueda en Facebook con las palabras "tuberculosis", "TBC" y "tisis", encontrando 207 grupos. De estos 71 fueron públicos, y de estos 15 tuvieron su contenido en español. 
De los 15 grupos, 10 fueron grupos de apoyo, $3 \mathrm{de}$ sensibilización y 2 de promoción. En la mayoría de grupos, el creador se identifica como proveniente de Perú o México. Estos resultados son esperables, dado que dichos países tienen alta incidencia de tuberculosis y se encuentran entre los países de habla hispana con mayor número de usuarios en Facebook ${ }^{6,7}$.

Solo tres grupos pertenecían a una institución de salud: el primero a la Universidad Federico Villareal (Perú), el segundo a la Universidad de El Salvador (El Salvador) y el tercero a la Universidad Tecnológica Pereira (Colombia).

Solo encontramos un grupo de apoyo que hubiese tenido un nuevo post en los últimos 30 días, en tanto que 4 tenían publicación entre 1 y 6 meses. Es decir, actualmente existe una carencia de grupos activos que brinden apoyo a pacientes con tuberculosis. Esto significa que se está perdiendo el gran potencial de apoyo que ofrecen las redes sociales virtuales, para influenciar positivamente en los pacientes con tuberculosis.

Resulta necesario realizar estudios que determinen el impacto de estos grupos de apoyo virtuales, así como sus características óptimas. Además, es recomendable que las entidades de salud creen y administren grupos abiertos, en los cuales los pacientes puedan encontrar apoyo e información veraz².

Marco Orellana-Presentacion $n^{1, a}$,
Claudia Benavides-Luyo ${ }^{1, a}$, Alvaro Taype-Rondan ${ }^{2, b}$
${ }^{1}$ Facultad de Medicina Humana, Universidad Ricardo
Palma, Lima, Perú.
${ }^{2}$ CRONICAS Centro de Excelencia en Enfermedades
Crónicas, Universidad Peruana Cayetano Heredia,
Lima, Perú.
${ }^{a}$ Estudiante de Medicina.
${ }^{b}$ Médico-Cirujano.

\section{Referencias}

1. Tuberculosis Organización Mundial de la Salud Lima-Perú: Organización Mundial de la Salud; 2015 [cited 201515 de Diciembre]. Available from: http://who.int/mediacentre/ factsheets/fs104/es/.

2. Respira vida, juntos contra la tuberculosis. Plan de Comunicación. Ministerio de Salud - Lima, Perú: 2011.

3. Bender JL, Jimenez-Marroquin MC, Jadad AR; Seeking Support on Facebook: A Content Analysis of Breast Cancer Groups. J Med Internet Res 2011;13(1):e16.

4. Leis Á, Mayer MÁ, Torres Niño J, Rodríguez-González A, Suelves JM, Armayones M. Grupos sobre alimentación saludable en Facebook: características y contenidos. Gaceta Sanitaria 2013; 27 (4): 355-7.

5. De la Torre-Díez I, Díaz-Pernas FJ, Antón-Rodríguez M. A content analysis of chronic diseases social groups on Facebook and Twitter. Telemedicine and e-Health 2012; 18 (6): 404-8.

6. Wikimedia. Facebook by countries Wikimedia Commons2011 [cited 2011 Julio 24]. Available from: https:// commons.wikimedia.org/wiki/File:Facebook_by_countriespng2.png.

7. La tuberculosis en la región de las Américas: Reporte Regional 2012, Epidemiología, Control y Financioamiento. Organización Panamericana de la Salud-Organización Mundial de la Salud, 2012.

Financiamiento: El presente estudio ha sido autofinanciado.

Correspondencia a:

Nombre: Marco Orellana-Presentacion

Dirección: Sector 3 Grupo 7 manzana I lote 24,

Villa el Salvador, Lima, Perú.

E-mail: marcoantorellypre@gmail.com 